\title{
Angiomotin promotes the malignant potential of colon cancer cells by activating the YAP-ERK/PI3K-AKT signaling pathway
}

\author{
YAN ZHANG ${ }^{1,2}$, JUN YUAN $^{3}$, XINLI ZHANG ${ }^{4}$, FUTANG YAN $^{3}$, MINGGANG HUANG $^{2}$, \\ TAO WANG $^{5}$, XIAO ZHENG ${ }^{3}$ and MING ZHANG ${ }^{1}$ \\ ${ }^{1}$ Department of Medical Imaging, The First Affiliated Hospital of Xi'an Jiaotong University College of Medicine, \\ Xi'an, Shaanxi $710061 ;{ }^{2} \mathrm{CT}$ Department, ${ }^{3}$ Department of Clinical Laboratory, ${ }^{4}$ Radio Immunity Center and \\ ${ }^{5}$ Department of Radiology, Shaanxi Provincial People's Hospital, Xi'an, Shaanxi 710068, P.R. China
}

Received April 17, 2016; Accepted June 7, 2016

DOI: $10.3892 /$ or.2016.5194

\begin{abstract}
Colorectal cancer (CRC) is a leading cause of cancer-related deaths with an increasing incidence in China. The aberrant expression of angiomotin (AMOT) has been confirmed in a variety of tumors and can interact with Yes-associated protein (YAP) to either promote or suppress the progression of cancer. Unfortunately, its role in CRC remains poorly elucidated. Herein, higher levels of AMOT were observed in CRC cell lines. Upregulation of AMOT in LoVo cells markedly increased cell proliferation and apoptotic resistance to 5-fluorouracil. Moreover, its increase also promoted cell invasion and migration. Simultaneously, AMOT silencing markedly attenuated the growth and metastatic potential of HCT116 cells. Notably, AMOT upregulation promoted the activity of YAP by decreasing the expression of phosphorylated YAP and YAP in the cytoplasm and increasing YAP levels in the nucleus. Further mechanistic analysis corroborated that transfection with YAP siRNA notably diminished cell growth, invasion and migration in the AMOT-overexpressing LoVo cells. Additionally, upregulation of AMOT induced the activation of the ERK and AKT pathways by YAP expression, both associated with the development of CRC. Collectively, these results suggest that AMOT may function as an oncogene in the progression of CRC by activating the YAP-ERK/PI3K-AKT signaling pathway. Therefore, this study presents a promising therapeutic target for CRC.
\end{abstract}

Correspondence to: Dr Ming Zhang, Department of Medical Imaging, The First Affiliated Hospital of Xi'an Jiaotong University College of Medicine, 277 Yanta West Road, Xi'an, Shaanxi 710061, P.R. China

E-mail: mingzhangpr@163.com

Key words: colorectal cancer, angiomotin, growth, invasion, migration, Yes-associated protein, ERK/AKT signaling pathway

\section{Introduction}

Colorectal cancer (CRC) is one of the most common gastrointestinal tract malignant carcinomas. It ranks as the third-leading cause of cancer-related deaths around the world (1). The increasing incidence and mortality rate of CRC has become a primary health concern in China (2). Currently, most patients with CRC are cured with surgical removal of the tumor, but this is not indicated for metastatic CRC patients. Hence, further research to elucidate the molecular mechanisms underlying CRC cell metastasis and a search for new targets are urgently needed for the effective treatment of CRC.

Angiomotin (AMOT) was initially identified as an angiostatin-binding protein and belongs to the motin family, which includes AMOT (p80 and p130 isoforms), AMOT-like protein 1 (AMOTL1) and AMOTL2 (3). AMOT is characterized by a C-terminal PDZ-binding motif and conserved coiled-coil domain and often exists in two different splicing isoforms, p80-AMOT and p130-AMOT. In contrast to p80-AMOT, there is an extended $\mathrm{N}$-terminal domain in p130-AMOT (4). Previous studies have suggested that AMOT can enhance endothelial cell motility and tube formation, implying a critical role in angiogenesis $(3,5)$. Recently, increasing attention has shed light on the role of AMOT in the pathogenesis of cancer $(6,7)$. Emerging evidence has corroborated an abnormal expression of AMOT in several types of cancer, such as breast cancer, lung cancer and renal carcinoma (7-10). AMOT has been proven to be highly expressed in breast cancer $(8,11)$. Notably, its downregulation obviously suppressed breast cancer cell proliferation and invasiveness by regulating the Hippo/Yes-associated protein (YAP) pathway (8). Additionally, AMOT exhibits a pro-proliferation role in renal cell carcinoma by retaining nuclear YAP, a common oncogenic gene (7). In contrast to the oncogenic role of AMOT, the antineoplastic function of AMOT has been observed in the progression of lung carcinoma by inhibiting cell growth and metastasis (9). To date, the functional role of AMOT in the development of CRC remains poorly elucidated.

In this study, we aimed to investigate the expression of $\mathrm{AMOT}$ and its function in cell growth, invasion and migration. Moreover, the potential molecular mechanism involved was also explored. 


\section{Materials and methods}

Antibodies. Primary antibodies against human YAP, phospho-AKT (p-AKT), AKT, ERK, phospho-ERK (p-ERK), $\mathrm{N}$-cadherin and E-cadherin were purchased from Cell Signaling Technology, Inc. (Danvers, MA, USA). Antibodies against cyclin D1, MMP-2, MMP-9, and GAPDH were obtained from Santa Cruz Biotechnology, Inc. (Dallas, TX, USA). The anti-tubulin and anti-AMOT antibodies were purchased from Abcam (Cambridge, UK). Rabbit polyclonal antibodies to human Bax and Bcl-2 were obtained from Sigma-Aldrich (St. Louis, MO, USA).

Cell culture. Human CRC cell lines LoVo, SW620, HT29 and HCT116 were obtained from the American Type Culture Collection (ATCC; Manassas, VA, USA). Normal human colon mucosal epithelium cell line NCM460 was ordered from the Incell Corporation, LLC (San Antonio, TX, USA). The CRC cells were all maintained in Dulbecco's Modified Eagle's medium (DMEM) supplemented with $10 \%$ fetal bovine serum (FBS), $100 \mathrm{U} / \mathrm{ml}$ penicillin and $100 \mu \mathrm{g} / \mathrm{ml}$ streptomycin. The NCM460 cells were cultured in an M3F base medium containing 10\% FBS. All cells were incubated at $37^{\circ} \mathrm{C}$ in a $5 \% \mathrm{CO}_{2}$ incubator (Life Technologies, Baltimore, MD, USA).

AMOT knockdown and overexpression. The knockdown and overexpression of AMOT in LoVo and HCT116 cells were performed using the lentiviral expression system provided by GeneChem Co., Ltd. (Shanghai, China). For AMOT overexpression and inhibition, the lentiviral vectors for human AMOT small hairpin RNA (shRNA) and vectors expressing AMOT (LV-AMOT) were constructed, packed and purified by GeneChem Co., Ltd. RNA interference sequences were used as follows: sh-AMOT-1, 5'-TGCAGAGATGGTGGAATAT-3'; sh-AMOT-2, 5'-ACACATCGAAATCCGAGAT-3'; the scrambled shRNA (sh-NC), 5'-TTCTCCGAATGTGTCACGT-3'. For transfection, cells were plated into 24-well plates. To increase the expression of AMOT, LoVo cells were transfected with LV-AMOT or control lentivirus ( $\mathrm{Lv}-\mathrm{NC})$ in accordance with the manufacturer's instructions. The lentivirus encoding AMOT shRNA or sh-NC was introduced into HCT116 cells. Approximately $8 \mathrm{~h}$ later, cells were incubated with fresh DMEM. After a 48-h incubation, the stable clones were selected for treatment with puromycin for 3 weeks. The efficacy of the lentivirus transfection was evaluated by western blotting.

RNAi-mediated silencing of YAP. To silence the expression of YAP in LoVo cells, small interfering RNA (siRNA) targeting human YAP and scramble siRNA (siR-con) were designed and synthesized by Shanghai GenePharma Co., Ltd. (Shanghai, China). The siRNA sequences were siR-YAP, 5'-GGUGAUAC UAUCAACCAAATT-3'; siR-con, 5'-CCUACGCCACCAAUU UCGU-3'. After seeding into 6-well plates for $12 \mathrm{~h}$, the cells were transfected with the siRNA duplexes $(100 \mathrm{nM})$ aforementioned, using Lipofectamine 2000 (Invitrogen Life Technologies Carlsbad, CA, USA) according to the manufacturer's instructions. At $48 \mathrm{~h}$ post-transfection, the transfection efficiency was assessed by western blotting.
Real-time quantitative RT-PCR ( $q R T-P C R$ ). To quantify the mRNA levels of AMOT, total RNA from the indicated cells was extracted using RNAiso Plus (Takara Bio Inc., Otsu, Japan) according to the manufacturer's instructions. Then, the obtained RNA was reversely transcribed into the first strand cDNA using the High-Capacity cDNA Reverse Transcription kits (Applied Biosystems, Foster City, CA, USA). The specimens were then subjected to RT-PCR with the specific primers for AMOT (sense, 5'-CCAGAATATCCCTTCAAG-3'; antisense, 5'-GAGTTCCTGGCTGACAAT-3'). qRT-PCR was performed with a final volume of $20 \mu 1$ according to the guidelines of the SYBR Premix Ex Taq ${ }^{\mathrm{TM}}$ II kit (Takara Bio Inc.). Human GAPDH was applied as the endogenous control. The relative expression of the target genes was calculated using the $2^{-\Delta \Delta \mathrm{Ct}}$ method.

Western blotting. Cells from the aforementioned samples were lysed with RIPA buffer, and the extracted protein concentrations were quantified using the BCA protein assay kit (Beyotime Institute of Biotechnology, Haimen, China). Then, $30 \mu \mathrm{g}$ of lysates was separated on SDS-PAGE and electroblotted onto PVDF membranes (Millipore, Bedford, MA, USA). After blocking the non-specific binding with 5\% nonfat milk in TBST buffer, the membranes were incubated with the primary antibodies against human AMOT, PCNA, ERK, p-ERK, p-AKT, AKT, cyclin D1, Bcl-2, Bax, MMP-2, MMP-9, N-cadherin, E-cadherin and YAP at $37^{\circ} \mathrm{C}$ for $1 \mathrm{~h}$. After 3 washes with TBST, a secondary antibody conjugated to horseradish peroxidase (HRP) was added for a further 1-h incubation. The immunoreactive bands were visualized using an ECL detection reagent (Beyotime Institute of Biotechnology). The band intensities were quantified by Quantity One software (Bio-Rad Laboratories, Inc., Hercules, CA, USA).

Immunoprecipitation assay. LoVo cells from the different treatments were harvested and lysed on ice for $20 \mathrm{~min}$ with lysis buffer, followed by centrifugation. For immunoprecipitation, $10 \mu \mathrm{g}$ of anti-YAP antibodies were pre-treated with $50 \mu \mathrm{l}$ protein A-Sepharose beads (Invitrogen Life Technologies) in $500 \mu \mathrm{l}$ of lysis buffer at $4^{\circ} \mathrm{C}$ for $15 \mathrm{~min}$. Then, the aforementioned mixture was added into the lysates at a volume of $500 \mu \mathrm{l}$ for a 1-h incubation. After washing with ice-cold lysis buffer, the formed immunocomplex was eluted by heating for $5 \mathrm{~min}$ at $95^{\circ} \mathrm{C}$. The immunoprecipitated proteins were separated by SDS-PAGE, then subjected to immunoblotting with the specific antibodies against AMOT.

Cell viability as detected by the 3-14,5-dimethylthiazolyl2-yl)-2,5-diphenyltetrazolium bromide (MTT) assay. Cell proliferation was detected using the MTT assay. Briefly, cells were seeded into a 96-well plate and incubated for 1-4 days after transfection. Then, the culture medium was removed and replaced with fresh medium including $5 \mathrm{mg} / \mathrm{ml}$ MTT (SigmaAldrich). After further incubation for $5 \mathrm{~h}$ at $37^{\circ} \mathrm{C}$, the remaining supernatant was discarded and $200 \mu \mathrm{l}$ of DMSO was introduced to dissolve the formed crystal formazan. The color reaction was detected by measuring the absorbance at $570 \mathrm{~nm}$ with an enzyme immunoassay analyzer (Bio-Rad Laboratories, Inc.). 
Measuring cell apoptosis by flow cytometric analysis. The cell apoptosis ratio was evaluated using an Annexin V-FITC/PI Apoptosis Detection kit (Beyotime Institute of Biotechnology). At $48 \mathrm{~h}$ after transfection, cells were collected by trypsinization and suspended in $500 \mu \mathrm{l}$ of binding buffer. Then, $10 \mu \mathrm{l}$ Annexin V-FITC and $5 \mu \mathrm{l}$ PI (Sigma-Aldrich) were added for a 15-min incubation at room temperature in the dark. The samples were subjected to flow cytometry (FACScan; BD Biosciences, San Jose, CA, USA) in a device equipped with CellQuest software to assess the apoptotic cells.

Migration and invasion assays. Cell invasion and migration assays were performed with or without Matrigel-coated inserts in the Transwell chambers (Millipore) according to the manufacturer's instructions. After treatment with the indicated conditions, cells in $200 \mu 1$ serum-free medium were seeded into the upper chamber. The medium including 10\% FBS was used as a chemoattractant to add into the lower chamber. Approximately $24 \mathrm{~h}$ later, the non-invading or non-migrated cells were removed by scrapping with a cotton swab. After fixation with $100 \%$ methanol, the invasive or migratory cells were stained with $0.5 \%$ crystal violet (Sigma-Aldrich) for $15 \mathrm{~min}$ at room temperature. The number of cells that had migrated or invaded through the membrane was quantified by counting the cells from at least three random fields with an inverted microscope.

Statistical analysis. Data were obtained from at least three independent experiments and are presented as the mean $\pm \mathrm{SD}$. All data analysis was carried out using SPSS 16.0 software (SPSS, Inc., Chicago, IL, USA). The statistical comparisons among the groups were evaluated using a Student's t-test and ANOVA. $\mathrm{P}<0.05$ was defined as statistically significant.

\section{Results}

Upregulation of AMOT in CRC cell lines. In order to explore the function of AMOT in the progression of CRC, a preliminary cohort study was performed in a series of CRC cell lines. In contrast to the NCM460 normal colon epithelium cell line, the mRNA levels of AMOT were markedly higher in the four examined CRC cell lines (Fig. 1A). Concomitantly, western blotting showed the notable increase of AMOT protein levels in CRC cells when compared to NCM460 cells (Fig. 1B).

Effect of AMOT expression on CRC cell growth. To dissect the effect of AMOT in CRC cells, AMOT protein levels were effectively increased in LoVo cells by LV-AMOT transfection (Fig. 2A). An MTT assay corroborated that overexpression of AMOT markedly increased the proliferation rate of the LoVo cells (Fig. 2B), concomitant with a similar increase in the expression of the PCNA protein, a common marker for cell proliferation (Fig. 2C). To further evaluate the role of AMOT overexpression on cell apoptosis, 5-fluorouracil (5-FU) was added to induce low basal apoptosis levels. As shown in Fig. 2D, AMOT upregulation obviously suppressed 5-FU-induced cell apoptosis from 29.87 to $16.94 \%$. To better investigate the function of AMOT in CRC cell growth, we constructed the stably AMOT-silenced cell line HCT116 by LV-AMOT shRNA transfection (Fig. 2E). In
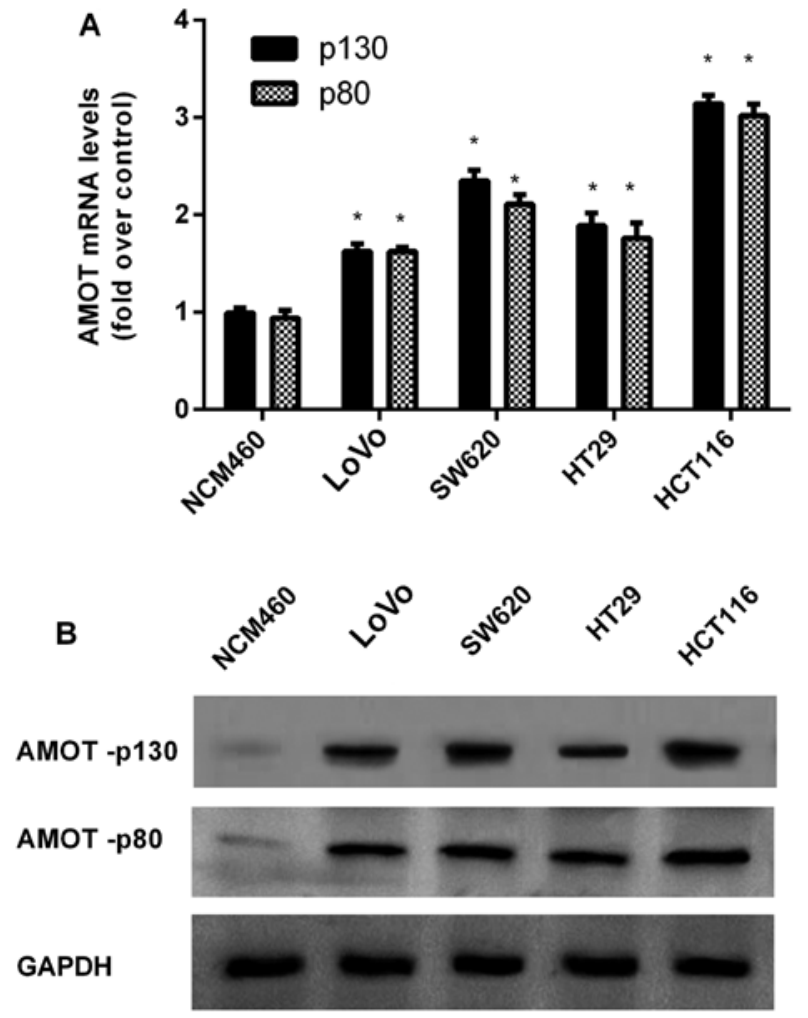

Figure 1. Expression of AMOT in CRC cell lines. (A) The mRNA levels of AMOT in four CRC cell lines (including Lovo, SW620, HT29 and HCT116) and a normal human colon mucosal epithelium cell line (NCM460) were detected by qRT-PCR. (B) The protein levels of AMOT were determined by western blotting. " $\mathrm{P}<0.05$ vs. NCM460. AMOT, angiomotin; CRC, colorectal cancer.

contrast, knockdown of AMOT expression in HCT116 cells markedly dampened the cell proliferation ability (Fig. 2F), accompanied by a corresponding decrease in PCNA expression (Fig. 2G). Compared to the control or sh-NC groups, AMOT silencing markedly increased the apoptotic ratio to $15.87 \%$ (Fig. $2 \mathrm{H}$ ). Collectively, these results indicate that AMOT promotes CRC cell growth and protects CRC cells against apoptosis.

AMOT promotes CRC cell invasion, migration and epithelial-mesenchymal transition (EMT). Based on the pro-growth role of AMOT in CRC cell growth, we further defined its effects on CRC cell metastatic potential by detecting cell invasion, migration and EMT. As shown in Fig. 3A, LV-AMOT-treated LoVo cells exhibited a statistically significant increase in cell invasion, compared with the control groups. Furthermore, an increase in AMOT markedly enhanced the number of LoVo cells migrating through the membrane (Fig. 3B). EMT is widely accepted as a major contributor to cancer cell metastasis. As expected, AMOT overexpression also reduced the expression of epithelial marker E-cadherin and increased the expression of mesenchymal marker N-cadherin (Fig. 3C). To further corroborate the aforementioned effects, the expression of AMOT was knocked down by LV-AMOT shRNA treatment. Blocking AMOT expression significantly antagonized the invasion ability of HCT116 cells (Fig. 3D). Simultaneously, the number of migrating HCT116 cells was also notably attenuated when cells were transfected with LV-AMOT 

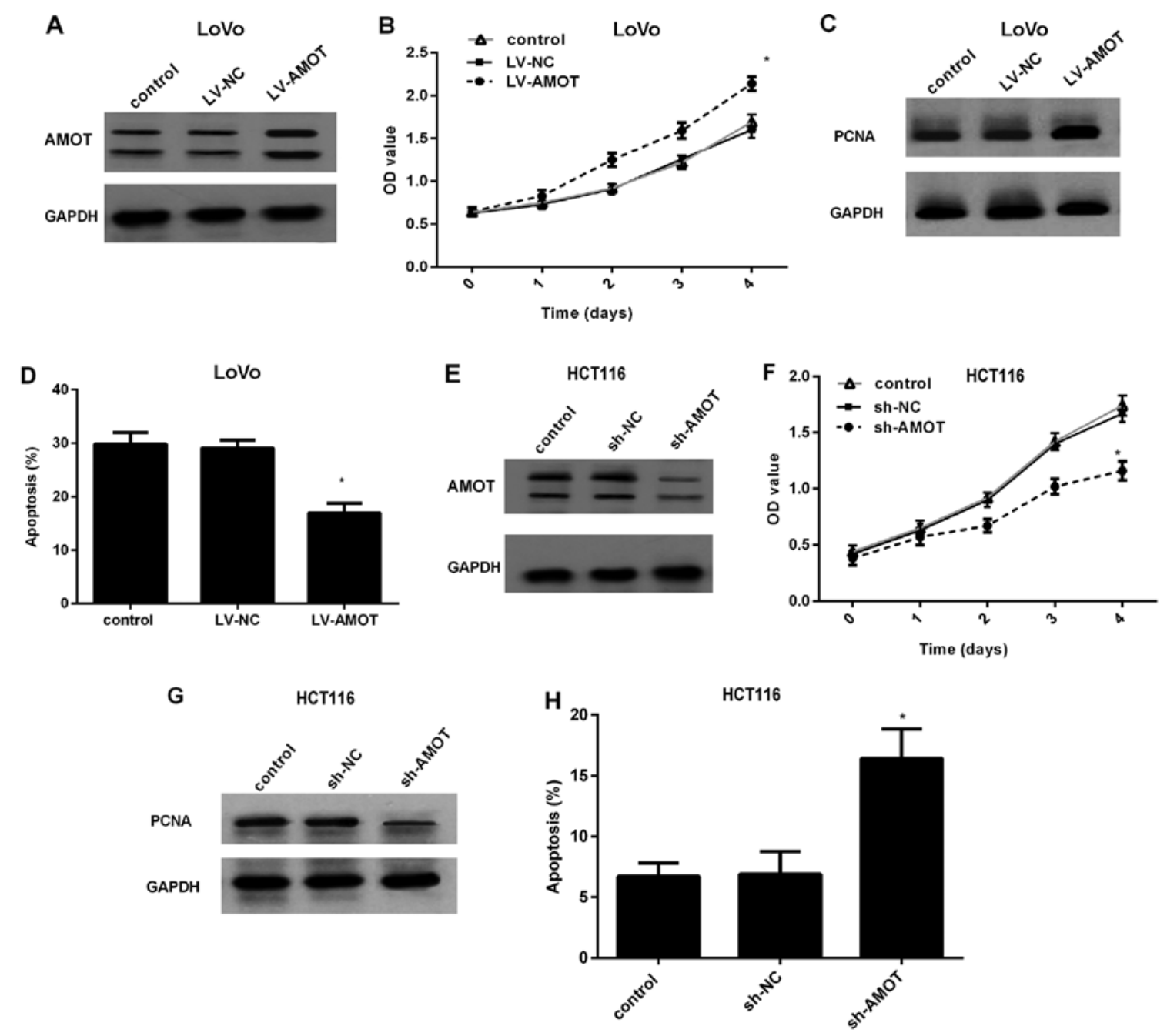

Figure 2. Effects of AMOT in CRC cell proliferation and apoptosis. (A) Following treatment with LV-AMOT, the protein levels of AMOT were evaluated by western blotting in Lovo cells. (B) An MTT assay was performed to detect cell proliferation at the indicated time-points (0-4 days). (C) The expression of PCNA was analyzed by western blotting in LV-AMOT-transfected LOVO cells. (D) The effect of AMOT upregulation on cell apoptosis was determined by flow cytometry. (E) HCT116 cells were infected with LV-AMOT shRNA. The knockdown efficiency of AMOT was evaluated by western blotting. (F and G) Effects of AMOT downregulation on cell proliferation and PCNA levels. $(\mathrm{H})$ The percentage of apoptotic cells in AMOT-silenced cells. "P<0.05 vs. control and NC groups. AMOT, angiomotin; CRC, colorectal cancer; shRNA, small hairpin RNA.

shRNA (Fig. 3E). Notably, downregulation of AMOT inhibited EMT by increasing the expression levels of E-cadherin and decreasing the levels of N-cadherin (Fig. 3F).

Upregulation of AMOT induces the activation of YAP by increasing the nuclear localization of YAP. AMOT has been reported to interact with YAP to stimulate its activity (7). Upon dephosphorylation, YAP is activated and then translocated to the nucleus to exert its oncogenic potential by activating downstream signaling (12). We further clarified the relationship between AMOT and YAP in CRC. In HCT116 cells, an antibody against YAP efficiently knocked down AMOT (Fig. 4A). To explore the effect of AMOT on YAP activity, the expression levels of phosphorylated YAP (p-YAP) were analyzed. As shown in Fig. 4B, p-YAP levels were mitigated in AMOT-overexpressing HCT116 cells (Fig. 4B). Moreover, upregulation of AMOT reduced the expression of cytoplasmic YAP (Fig. 4C). Notably, treatment with LV-AMOT significantly increased the relative expression of nuclear YAP (Fig. 4D). These data indicated that AMOT induces the activity of YAP in CRC cells.
YAP is required for the oncogenic effect of AMOT overexpression in CRC cells. To elucidate the underlying molecular mechanism involved in the AMOT-induced oncogenic effect in HCT116 cells, we explored the role of YAP during this process. Western blotting illustrated the knockdown of YAP in HCT116 cells transfected with YAP siRNA (Fig. 5A). A function assay validated that silencing of YAP markedly antagonized the pro-proliferation effect of AMOT overexpression in HCT116 cells (Fig. 5B). Furthermore, AMOT upregulation notably enhanced cell resistance to 5-FU-induced apoptosis, which was obviously attenuated following YAP downregulation (Fig. 5C). Concomitantly, knockdown of YAP significantly attenuated the invasion (Fig. 5D) and migration (Fig. 5E) of HCT116 cells triggered by AMOT upregulation. Collectively, these results revealed that AMOT may promote $\mathrm{CRC}$ cell growth, invasion and migration mainly through YAP.

Increase in AMOT activates the ERK/PI3K-AKT signaling pathway in a manner mediated by YAP. Abnormal activation of 

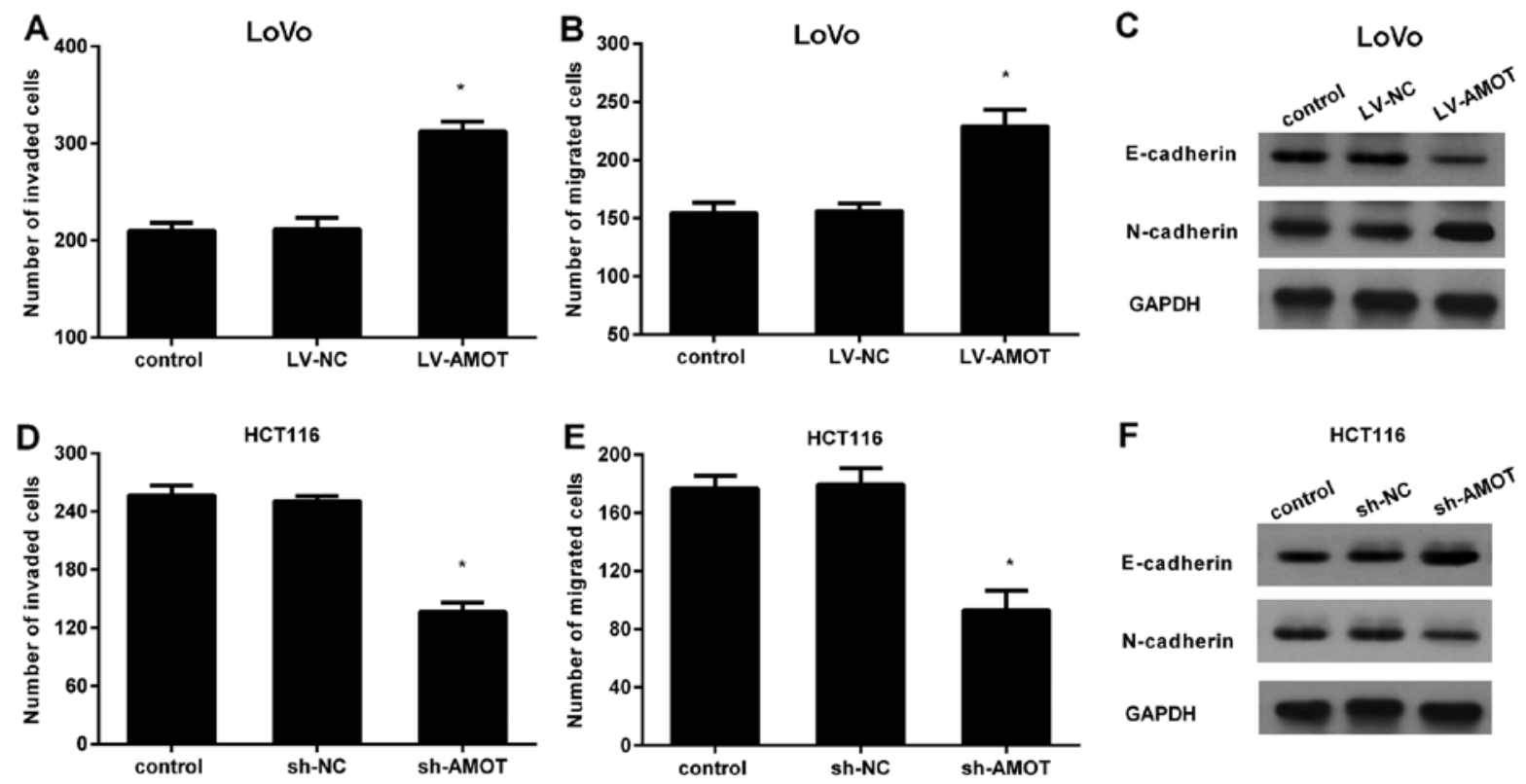

Figure 3. AMOT enhances CRC cell invasion, migration and EMT in vitro. (A and B) After the overexpression of AMOT in LOVO cells, a Transwell assay was utilized to assess cell invasion and migration. (C) Expression levels of E-cadherin and $\mathrm{N}$-cadherin were determined to analyze the effect of AMOT upregulation on EMT. (D-F) The effect of AMOT knockdown on (D) HCT116 cell invasion, (E) migration and (F) EMT. "P<0.05. AMOT, angiomotin; CRC, colorectal cancer; EMT, epithelial-mesenchymal transition.

\section{A}

IP:

YAP

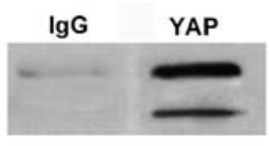

YAP

Input: AMOT

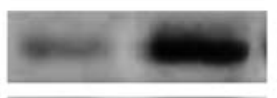

c

C

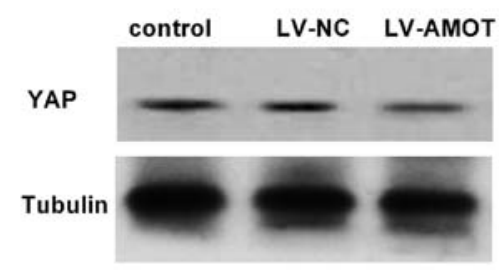

B

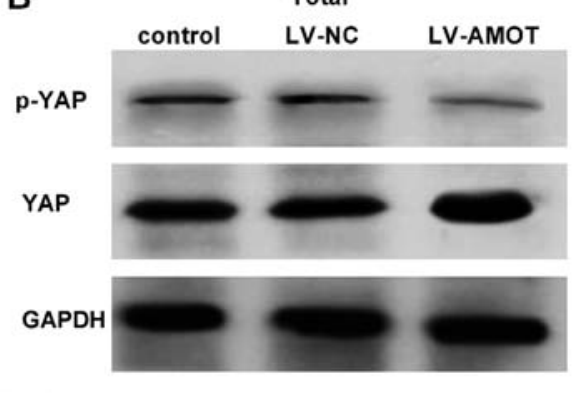

D

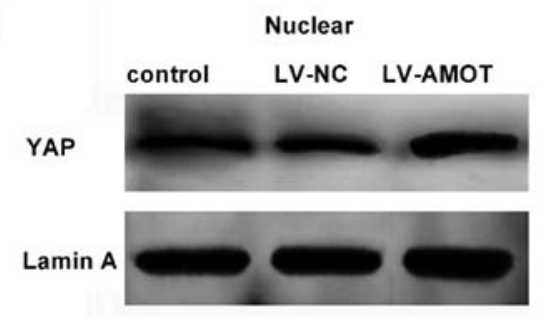

Figure 4. Increase of AMOT induces the activation of YAP. (A) Cell lysates from the HCT116 cells were immunoprecipitated with anti-YAP antibody or control IgG. Then, western blotting was used to analyze the endogenous interaction between AMOT and YAP. (B) Expression levels of p-YAP and total YAP were detected by western blotting. GAPDH was introduced as a loading control. (C) Expression of YAP in cytoplasmic fraction in the HCT116 cells. Tubulin was used to normalize gene expression. (D) Western blotting was performed to measure YAP expression in the nuclear fraction. Lamin A was used as an internal control. AMOT, angiomotin; YAP, Yes-associated protein; IP, immunoprecipitation; IB, immunoblotting.

the ERK and PI3K/AKT pathways has been reported in various carcinomas and they play critical roles in carcinogenesis by regulating cell proliferation, invasion and migration (13-16). To further elucidate the mechanism involved in AMOT-mediated oncogenic potential, both of these two pathways were explored further. In line with our hypothesis, overexpression of AMOT markedly induced the expression of p-ERK and p-AKT, but not the total ERK and AKT (Fig. 5F). A previous study confirmed that YAP can transcriptionally activate the PI3K/AKT and ERK signaling pathways in human cutaneous squamous cell cancer (12). Further analysis of the mechanism demonstrated that silencing of YAP expression markedly attenuated AMOT-induced expression of p-ERK and p-AKT (Fig. 5G), accompanied by the decrease in the subsequent expression of cyclin D1, Bcl-2 and the increase in the expression of Bax (Fig. 5H). Additionally, the downstream increases in MMP-2 and MMP-9 levels in AMOT-elevated cells were obviously attenuated after YAP siRNA transfection (Fig. 5I). 
A

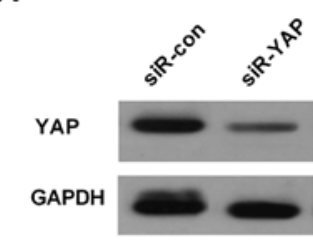

D

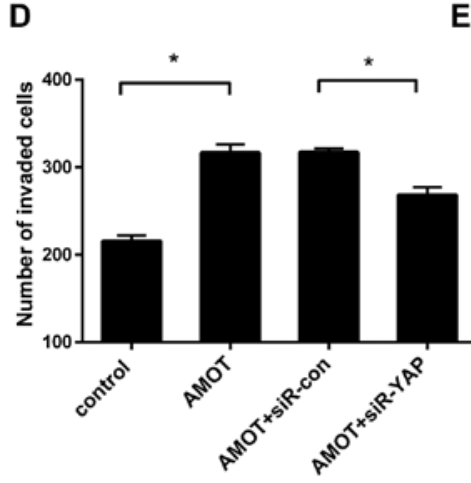

G

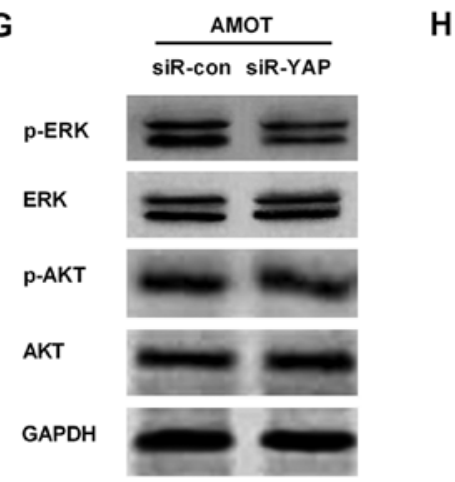

E

H
B
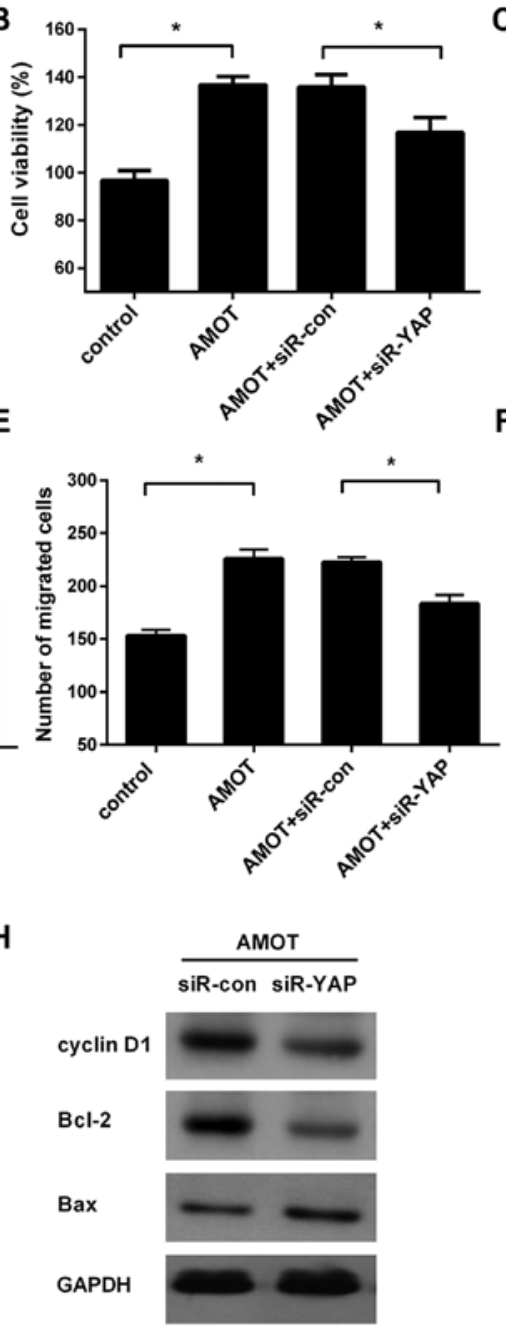

$\mathbf{F}$
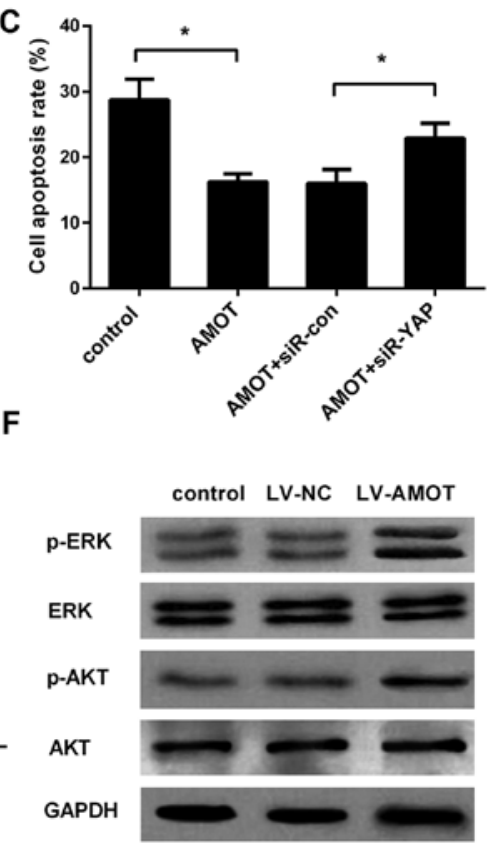

I

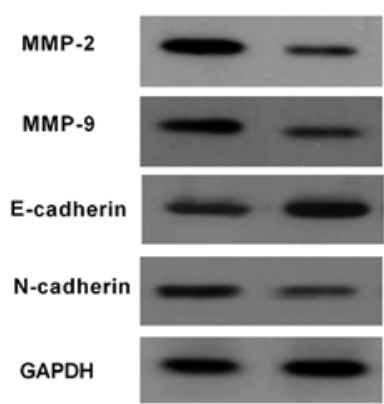

Figure 5. AMOT promotes CRC cell growth, invasion and migration through the YAP-ERK/PI3K-AKT signaling pathway. HCT116 cells were transfected with YAP siRNA, prior to infection with LV-AMOT. (A) The efficacy of YAP silencing was analyzed. (B-E) Evaluation the effects of YAP knockdown on cell viability, apoptosis, invasion and migration triggered by AMOT overexpression. (F) After infection with LV-AMOT, the activation of the ERK and PI3K/AKT pathways was assessed by western blotting. (G) Effects of YAP silencing on AMOT-induced activation of the ERK and PI3K/AKT signaling pathways. (H) The corresponding expression of downstream effectors related to cell proliferation (cyclin D1, Bcl-2 and Bax). (I) After preconditioning with YAP siRNA, the expression of ERK and PI3K/AKT signaling proteins involved in cell metastatic potential (MMP-2, MMP-9, E-cadherin and N-cadherin) was monitored in AMOT-overexpressed HCT116 cells. "P<0.05. AMOT, angiomotin; CRC, colorectal cancer; YAP, Yes-associated protein; siRNA, small interfering RNA.

Consistently, YAP knockdown also inhibited AMOT-induced EMT by increasing E-cadherin expression and suppressing $\mathrm{N}$-cadherin levels. All of these results implied that AMOT could induce the activation of the YAP-ERK/PI3K-AKT signaling pathway.

\section{Discussion}

Recent studies have demonstrated the aberrant alteration of AMOT in the progression of cancer $(7,9,10)$. However, its role in carcinogenesis is controversial in various types of cancer. For example, AMOT exerts oncogenic effects in breast cancer by promoting cell proliferation and invasion through the Hippo/YAP pathway (8). Notably, knockout of AMOT in the livers of mice led to reduced hepatic 'oval cell' proliferation and tumorigenesis (10). Other evidence substantiates that knockdown of AMOT initiates lung cancer cell proliferation, migration, invasion and ultimately acts as a tumor suppressor in lung carcinoma progression by sequestering oncogenic YAP/TAZ (9). In the present study, the higher expression of AMOT was detected in CRC cells. Importantly, overexpression of AMOT promoted LoVo cell proliferation and resistance to 5-FU-induced apoptosis. Moreover, its upregulation also enhanced cell invasion, migration and EMT. Consistently, blocking AMOT expression consistently inhibited HCT116 cell growth and metastatic potential. Therefore, these findings suggest that AMOT may elicit the oncogenic function in the carcinogenesis of CRC.

Accumulating evidence has revealed that aberrant alteration of key components of the Hippo signaling pathway can result in uncontrolled cell growth and cancer progression $(17,18)$. As a major downstream transcription activator of the Hippo pathway, YAP can bind to several transcription factors to regulate the development of cancer. Indeed, 
high expression of YAP has been noted in a wide variety of cancers, including CRC $(12,19)$. However, YAP is inactivated and sequestered in the cytoplasm when phosphorylated by the kinase of the Hippo pathway (20). Once dephosphorylated, it becomes activated and translocates to the nucleus, where it can act as an oncogenic regulator for tumor cell growth, invasion and migration $(12,21)$. Recent studies confirmed the correlation between AMOT and YAP, however, this is controversial $(7,22)$. In the liver, AMOT can function as a YAP cofactor to prevent YAP phosphorylation and increase its activity toward a specific set of genes that facilitate tumorigenesis (10). In contrast to the aforementioned finding, AMOT can interact with YAP to sequester YAP in the cytoplasm, which finally mitigates the development of lung cancer (9). Whether AMOT stimulates or suppresses YAP activity in CRC remains undefined. In our study, AMOT interacted with YAP in HCT116 cells. More importantly, AMOT decreased the levels of p-YAP. Furthermore, overexpression of AMOT attenuated the expression of YAP in the cytoplasm and increased its levels in the nucleus. All of these data indicated that AMOT could promote YAP activity in CRC cells, implying a potential oncogenic function of AMOT in the progression of CRC.

It is believed that the progression and metastasis of malignant tumors are complicated processes involved in multiple cellular events, including cell growth, invasion and migration. Here, we demonstrated that AMOT upregulation enhanced CRC cell proliferation, apoptosis resistance, invasion, migration and EMT. Simultaneously, blocking AMOT expression also inhibited CRC cell growth and motility. As an oncogene, YAP is highly expressed in CRC and acts as a predictor of poor prognosis in CRC patients (23). Moreover, YAP mediated the high proliferation and metastasis in CRC cells. Notably, our study confirmed the increased activity and expression of YAP in AMOT-overexpressing CRC cells. All of these findings led us to speculate as to whether YAP is involved in the AMOT-mediated oncogenic function in CRC. To address this hypothesis, we silenced YAP expression. As expected, knockdown of YAP markedly attenuated the malignancy of cell growth, invasion and migration upon AMOT overexpression, indicating a critical role of YAP in the AMOT-triggered metastasis-promoting process of CRC.

The ERK and PI3K/AKT pathways are often simultaneously activated in various carcinomas and have therefore been the focus of numerous investigations in CRC $(13,14)$. It is widely accepted that both the ERK and the PI3K/AKT pathways stimulate a cascade of responses from cell survival to metastasis by their downstream effectors, which ultimately facilitates the development and progression of CRC (13-16). Results from previous studies demonstrated that AMOT upregulation increased the phosphorylation of ERK and AKT $(17,24)$. Notably, a recent study revealed that YAP contributed to the development of human cutaneous squamous cell cancer by activating the RAS-mediated AKT and ERK signaling pathways (12). Moreover, YAP overexpression was found to increase EMT in pancreatic cancer cells by activating the AKT cascade (25). YAP also enhanced the ERK/AKT signaling pathway in renal cell carcinoma cells (7). Does AMOT regulate the activation of the ERK/AKT pathway through YAP? To answer this question, we explored the effects of YAP silencing during this process. As we hypothesized, YAP knockdown significantly attenuated AMOT-induced activation of the ERK/AKT pathway, as well as the activation of their downstream targets associated with tumor cell growth and metastasis. These findings support the hypothesis that AMOT induces the activation of the AKT/ERK pathway through YAP, which facilitates the tumorigenesis of CRC.

In conclusion, this study confirmed the upregulation of AMOT in CRC cells. Notably, overexpression of AMOT promoted the cell growth and metastatic potential in CRC cells mainly by activating the YAP-ERK/AKT signaling pathway. Accordingly, our study may provide new insight concerning how AMOT acts as an oncogene in the progression of CRC, presenting a promising therapeutic agent against CRC.

\section{Acknowledgements}

Financial support was provided by a project supported by the Natural Science Basic Research Plan in Shaanxi Province of China (no. 2014JM2-8201) and the National High Technology Research and Development Program ('863' Program) (no. 2014AA022304).

\section{References}

1. Weitz J, Koch M, Debus J, Höhler T, Galle PR and Büchler MW: Colorectal cancer. Lancet 365: 153-165, 2005.

2. Hu F, Li D, Wang Y, Yao X, Zhang W, Liang J, Lin C, Ren J, Zhu L, Wu Z, et al: Novel DNA variants and mutation frequencies of $h M L H 1$ and $h M S H 2$ genes in colorectal cancer in the Northeast China population. PLoS One 8: e60233, 2013.

3. Troyanovsky B, Levchenko T, Månsson G, Matvijenko O and Holmgren L: Angiomotin: An angiostatin binding protein that regulates endothelial cell migration and tube formation. $\mathrm{J}$ Cell Biol 152: 1247-1254, 2001.

4. Ernkvist M, Aase K, Ukomadu C, Wohlschlegel J, Blackman R, Veitonmäki N, Bratt A, Dutta A and Holmgren L: p130-angiomotin associates to actin and controls endothelial cell shape. FEBS J 273: 2000-2011, 2006.

5. Bratt A, Birot O, Sinha I, Veitonmäki N, Aase K, Ernkvist M and Holmgren L: Angiomotin regulates endothelial cell-cell junctions and cell motility. J Biol Chem 280: 34859-34869, 2005.

6. Moleirinho S, Guerrant W and Kissil JL: The angiomotins - from discovery to function. FEBS Lett 588: 2693-2703, 2014.

7. Lv M, Li S, Luo C, Zhang X, Shen Y, Sui YX, Wang F, Wang X, Yang J, Liu P, et al: Angiomotin promotes renal epithelial and carcinoma cell proliferation by retaining the nuclear YAP. Oncotarget 7: 12393-12403, 2016.

8. Lv M, Lv M, Chen L, Qin T, Zhang X, Liu P and Yang J: Angiomotin promotes breast cancer cell proliferation and invasion. Oncol Rep 33: 1938-1946, 2015.

9. Hsu YL, Hung JY, Chou SH, Huang MS, Tsai MJ, Lin YS, Chiang SY, Ho YW, Wu CY and Kuo PL: Angiomotin decreases lung cancer progression by sequestering oncogenic YAP/TAZ and decreasing Cyr61 expression. Oncogene 34: 4056-4068, 2015.

10. Yi C, Shen Z, Stemmer-Rachamimov A, Dawany N, Troutman S, Showe LC, Liu Q, Shimono A, Sudol M, Holmgren L, et al: The p130 isoform of angiomotin is required for Yap-mediated hepatic epithelial cell proliferation and tumorigenesis. Sci Signal 6: ra77, 2013.

11. Zhang $\mathrm{H}$ and Fan Q: MicroRNA-205 inhibits the proliferation and invasion of breast cancer by regulating AMOT expression. Oncol Rep 34: 2163-2170, 2015.

12. Jia J, Li C, Luo S, Liu-Smith F, Yang J, Wang X, Wang N, Lai B, Lei T, Wang Q, et al: Yes-associated protein contributes to the development of human cutaneous squamous cell carcinoma via activation of RAS. J Invest Dermatol 136: 1267-1277, 2016.

13. Ye Q, Cai W, Zheng Y, Evers BM and She QB: ERK and AKT signaling cooperate to translationally regulate survivin expression for metastatic progression of colorectal cancer. Oncogene 33: 1828-1839, 2014. 
14. Huynh N, Liu KH, Baldwin GS and He H: P21-activated kinase 1 stimulates colon cancer cell growth and migration/invasion via ERK- and AKT-dependent pathways. Biochim Biophys Acta 1803: 1106-1113, 2010.

15. Zhao D, Sui Y and Zheng X: MiR-331-3p inhibits proliferation and promotes apoptosis by targeting HER 2 through the $\mathrm{PI} 3 \mathrm{~K} / \mathrm{Akt}$ and ERK1/2 pathways in colorectal cancer. Oncol Rep 35: 1075-1082, 2016.

16. Huang J, Che MI, Lin NY, Hung JS, Huang YT, Lin WC, Huang HC, Lee PH, Liang JT and Huang MC: The molecular chaperone cosmc enhances malignant behaviors of colon cancer cells via activation of Akt and ERK. Mol Carcinog 53 (Suppl 1): E62-E71, 2014

17. Harvey KF, Zhang X and Thomas DM: The Hippo pathway and human cancer. Nat Rev Cancer 13: 246-257, 2013.

18. Lin L, Sabnis AJ, Chan E, Olivas V, Cade L, Pazarentzos E, Asthana S, Neel D, Yan JJ, Lu X, et al: The Hippo effector YAP promotes resistance to RAF- and MEK-targeted cancer therapies. Nat Genet 47: 250-256, 2015.

19. Li H, Wang S, Wang G, Zhang Z, Wu X, Zhang T, Fu B and Chen G: Yes-associated protein expression is a predictive marker for recurrence of hepatocellular carcinoma after liver transplantation. Dig Surg 31: 468-478, 2014.

20. Zhao B, Lei QY and Guan KL: The Hippo-YAP pathway: New connections between regulation of organ size and cancer. Curr Opin Cell Biol 20: 638-646, 2008.
21. Dai XY, Zhuang LH, Wang DD, Zhou TY, Chang LL, Gai RH, Zhu DF, Yang B, Zhu $\mathrm{H}$ and He QJ: Nuclear translocation and activation of YAP by hypoxia contributes to the chemoresistance of SN38 in hepatocellular carcinoma cells. Oncotarget 7: 6933-6947, 2016.

22. Zhao B, Li L, Lu Q, Wang LH, Liu CY, Lei Q and Guan KL: Angiomotin is a novel Hippo pathway component that inhibits YAP oncoprotein. Genes Dev 25: 51-63, 2011.

23. Wang L, Shi S, Guo Z, Zhang X, Han S, Yang A, Wen W and Zhu Q: Overexpression of YAP and TAZ is an independent predictor of prognosis in colorectal cancer and related to the proliferation and metastasis of colon cancer cells. PLoS One 8: e65539, 2013.

24. Ranahan WP, Han Z, Smith-Kinnaman W, Nabinger SC, Heller B, Herbert BS, Chan R and Wells CD: The adaptor protein AMOT promotes the proliferation of mammary epithelial cells via the prolonged activation of the extracellular signal-regulated kinases. Cancer Res 71: 2203-2211, 2011.

25. Yuan Y, Li D, Li H, Wang L, Tian G and Dong Y: YAP overexpression promotes the epithelial-mesenchymal transition and chemoresistance in pancreatic cancer cells. Mol Med Rep 13: 237-242, 2016. 